\title{
Digital education facing COVID - 19 pandemic: technological university experience
}

\author{
Oleg Pobegaylov*
}

Don State Technical University, 344002, Rostov-on-Don, Russia

\begin{abstract}
The article deals with the aspects of electronic and distance education under the COVID19 pandemic. It is noteworthy that there are common features in managing electronic and online education in higher school, as well as the problems of applying these formats of education. The tools of the educational process of digital education, the effectiveness of their use, the reaction of students and teachers to the new learning environment and technologies are described. The author argues that information and communication technologies are increasingly melting into various areas of education. This is facilitated by both external factors related to the widespread computerization of the society and the need for appropriate training of specialists, and internal factors related to the spread of modern digital technologies and software in school, the adoption of governmental and intergovernmental programs for the computerization of education, the emergence of the relevant digital experience of a large number of instructors. In most cases, the use of digital tools produces a real positive impact on the intensification of the work of the educators both in high school and university, as well as on the effectiveness of students' training, despite a number of shortcomings of the existing distant education system.
\end{abstract}

\section{Introduction}

The creation of digital economy is a global trend that dates back to the late 1990s, when for the first time global communication networks shaped up the market for digital products and services. From chain stores, contact-free payment systems and remote communication, the world has now moved to virtual reality and the digital sector of the economy [1].This is facilitated by both external factors related to the widespread computerization of the society and the need for appropriate training of specialists, and internal factors related to the spread of modern digital technologies and software in school, the adoption of governmental and intergovernmental programs for the computerization of education, the emergence of the relevant digital experience of a large number of instructors. In most cases, the use of digital tools produces a real positive impact on the intensification of the work of the educators both in high school and university, as well as on the effectiveness of students' training, despite a number of shortcomings of the existing distant education system.

*Corresponding author: georgy-2583@yandex.ru 


\section{Materials and methods}

Higher education in Russia did not adapt to the new economic realities straight away. For long, digitalization in universities was understood as a purely technical progress associated with the transition from the mechanical means of printing documents to digital ones, the development of the capabilities of presentational equipment, software that boosted the efficiency of using computational and graphic stuff in school and factory [1-3]. Due to the increasing activity of interaction between people, to the progress in the technical means of communication, a new type of culture is being formed - the culture of virtual communication. Describing this phenomenon, we may highlight its main properties: the non-linear way of existence of the communicating subject, the virtualization of public relations, the increasing part of social institutions that form the virtual communication space, etc.

Presently, the main focus and time of the educator's academic work is focused on providing their students with data, familiarizing them with information, transferring knowledge and forming their understanding. Our study reveals a vivid relationship between the effect of feedback and the effectiveness of distant learning (Fig. 1)

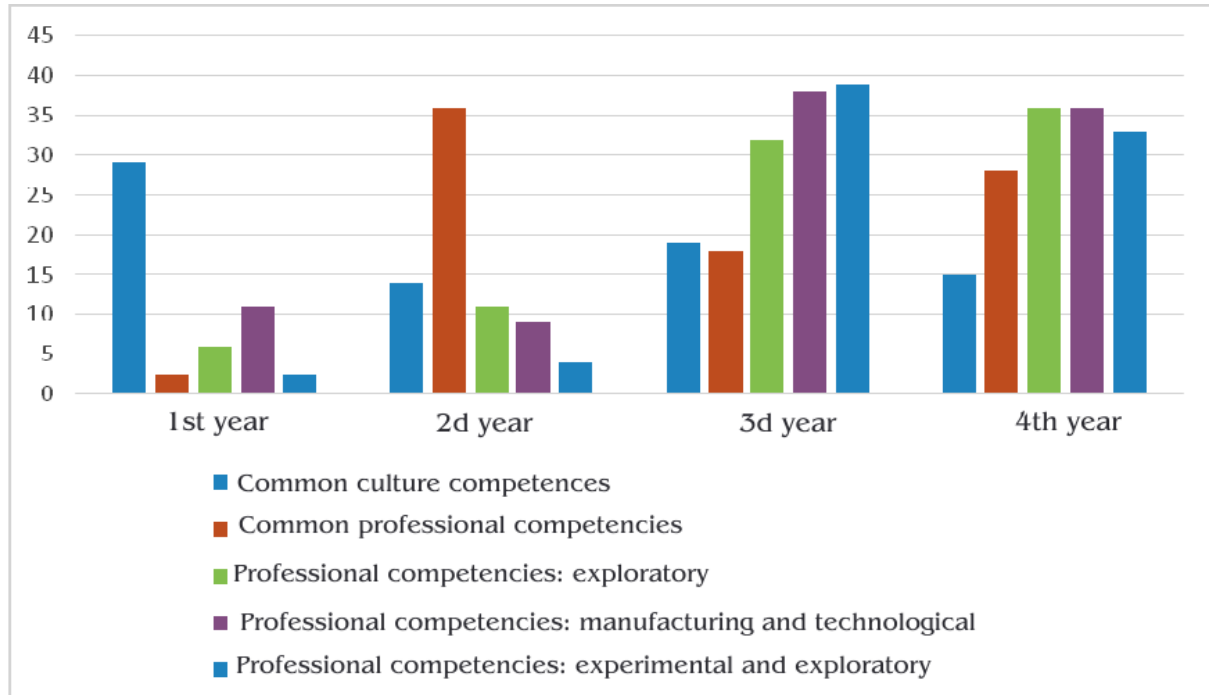

Fig. 1. Development of competencies in the specialty and master's programs in the directions 08.04.01 and 08.05.01 "Construction" as per cent of the total competence based on the results of tests performed in a period of distance learning

The competence development chart shows that the results of the competencies acquired during the period under study are quite low, and no competence has been achieved even at $50 \%$ of the possible maximum. At the same time, the distribution of acquisition among students shows other dependencies.

Hence, the number of students who completed the program amounts to $75.2 \%$ of the total number of students involved in the study. This suggests that the students have gained some knowledge in their professional field, but this knowledge requires further improvement to be fully professionally implemented.

These data can be compared to the research on the development of competencies in the study of subjects in the full-time classroom format. 


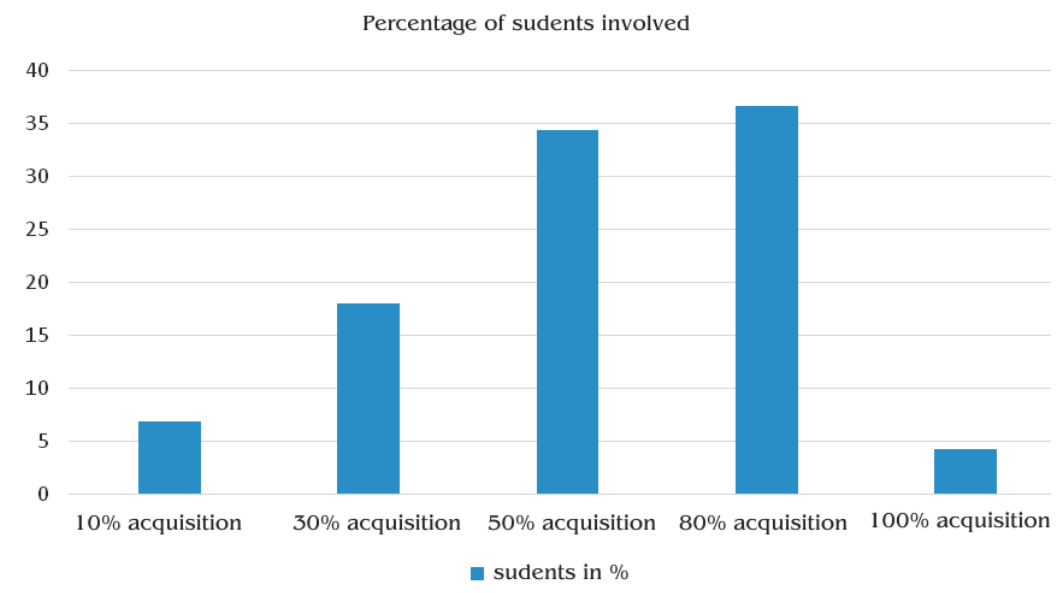

Fig.2. Acquisition of all of the competencies in the whole body of students in the specialty and master's programs in the directions 08.04.01 and 08.05.01 "Construction" in per cent of the total competence

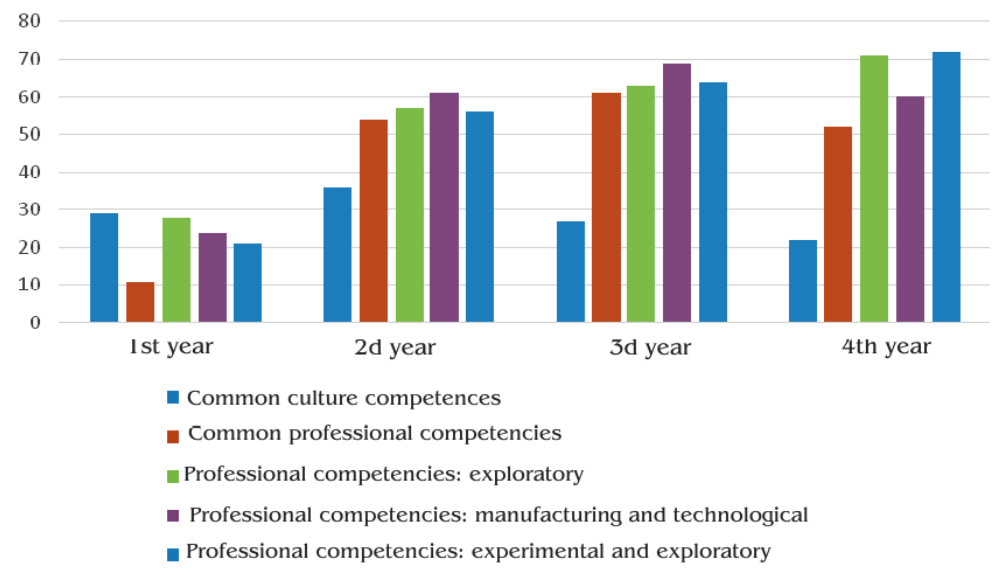

Fig. 3. Acquiring competences competencies in the specialty and master's programs in the directions 08.04.01 and 08.05.01 "Construction" in per cent of the total 100\% competence as per the results of tests done during the full-time classroom period

As our research shows, in full-time classroom training, the percentage of mastering competencies is over the average, save for the first academic year. The low percentage of training only shows the mastering of common cultural competencies.

The key problem of abrupt switching to distance learning in university was the psychological crisis caused by the drop in contacts with the outside world and selfisolation. The situation they faced in everyday life became common for work (full-time) [47]. The psychological state required for work and training has ceased to be prompted by the usual change of environment from home to school. The influence of the university as disciplining environment has ceased to consolidate the cognitive processes of participants in academic communication.

The psychological problem for instructors in the spring of 2020 was aggravated by the sharply declining physical activity. In the first two weeks, they had to stay long at the computer to prepare and mail educational packages, download regulatory and reference documents on these sites, in chats and social networks. Hence, there arose a single psycho- 
physiological problem of operating in the new environment, where physical fatigue from working with a computer developed in parallel with the growth of moral fatigue psychosomatic anxiety [8].

\section{Results}

A significant element of learning is to maintain feedback from the student to the instructor. With the total distance learning, this connection gets nearly lost, since the student does not meet with the instructor even at tests. In the video conference mode, you can answer some of the students' questions, but such feedback is severely compromised due to the lack of visual contact [9-11]. The similarity of this contact could be provided by conducting a video call conversation in a messenger, which was performed by some instructors of the Don State Technical University. With this method of providing feedback, at least three drawbacks are identified: the instructor spends a lot of time on individual conversations, while the other students do not hear the main part of the conversation and the learning element is not even brought to them indirectly; the student can read information from other devices during a video call conversation, and the instructor won't fully assess the student's knowledge of the issue discussed.

Among the positive effects of restrictive measures caused by to the spread of the COVID -19 , we should note the rapid development of online learning technologies by instructors, and, as many instructors claim, an increased attendance at online classes compared to offline ones. Even those instructors who did not initially rush to create online courses were now forced to do so, because they were challenged with a choice to create such courses and master the appropriate digital technologies, or to stop teaching at all. Students who combined their studies with work were now able to attend online classes due to the ease of connection and the lack of need to spend time to get to the place of study [12$15]$.

It is noteworthy that the total switchover of the whole body of students to online learning in the spring of 2020 still revealed more shortcomings than positive aspects. In addition to the psychological and psycho-physiological problems discussed above, the quality of training has suffered. It was revealed that not all types of classes and not all sciences can be taught online without a significant loss in the effectiveness of learning. If the disadvantage of the remote mode is only the lack of the effect of live communication, then to instill in students the necessary skills and abilities in online classes was only for those instructors who were digitally educated, who, as a rule, taught subjects related to the development of computer technologies. Laboratory classes and training practices turned out to be completely impossible to conduct.

Because of the deteriorating quality of education and the actual transfer of students from full-time to part-time format, the students of a few universities who are studying on a compensatory form of education and have paid for full-time education demanded a refund of their fees, since the part-time format is usually five times cheaper than that of full-time.

Many schools have stumbled upon the problem of using copyrights and related rights to the information technologies applicable for distance education, with the technical unavailability of the university material base to ensure sustainable online work due to the increased load on services that were previously used as teaching aids. For example, The SKIF platform at the Don State Technical University has failed to provide a high-quality support in the mass mode of distance learning: low quality of the provided signal, which led to bad sound, periodic lack of access to the platform for some students, and other shortcomings. This was further complicated by some general problems with communication 
which were aggravated in some remote areas due to the low performance of their telecommunications networks. It should be understood that many students who were forced to move from university dormitories to other accomodations, also in hard-to-reach areas, during the period of restrictions caused by the COVID-19, were almost completely deprived of the opportunity to attend online classes through digital communication channels.

On switching to the online format, the socially educational activities at the university are suffering greatly. Neither the curators nor the instructors can provide their educational influence in an online video class. More than that, of course, a significant part of educational work is carried out in the classroom, where the instructor turns into an educator, i.e. not only shares knowledge, but also educates students, introducing them to the proper code of conduct and culture practices, etc. Socially educational work, in addition, where introduced correctly, is performed comprehensively, i.e. students get involved in the scientific and methodological work of a corresponding department. The correct approach to educational work allows to pick out students who want to engage in research, identify young talents and improve academic performance in regular classes and supervisor student meetings. However, all this is impossible in the context of distance learning.

The nearly six-month experience of the instructors of the Don State Technical University in teaching students remotely has revealed the following types of interaction, the digitalization of which seems most promising.

Summing up, as recommendations for improving educational technologies in the context of digitalization, the authors offer:

1. Initially, when developing training based on individual plans, it is the online learning that takes over. Therefore, with this mode of training, digitalization should cover nearly the entire learning process, and meetings with instructors and scientific supervisors in full-time format can be made periodically just to clarify some most difficult issues of mastering subjects and current research, as well as (partially) to conduct intermediate testing in sciences, practical works and term projects.

It is possible to partially computerize the training of students in groups based on regular curricula. First of all, the experience of the Don State Technical University has shown that digital technologies are most successfully used at performing the final state examination. The presentation of the diploma works was successfully conducted at video conferences. As it turned out, this is no more difficult to organize than a normal shop meeting held remotely. Failures in the operation of the equipment during the state final examination both in June and December 2020 were rare. In only one per cent of all cases, it was necessary to postpone the presentation of a student's diploma work due to the malfunctioning communication equipment they were using to communicate with the examination board. Undoubtedly, the organization of the presentation of final diploma works requires a highly qualified secretary of the board. The secretary, a few days before the presentation of the final diploma, must properly check the performance of the student's equipment and their ability to use the relating software. To help make the work of the secretary easier, such checking can be done by the supervisors of the students' final diploma works.

In the totally digital format, it is possible to deliver lectures in humanities, technical and natural sciences; it is possible to partially handout lecture contents in digital format. During the lectures at video conferences at the Don State Technical University, students had trouble perceiving formulas and doing geometric tracing. To solve this problem, instructors are bound to send electronic handouts to students beforehand to make them get familiarized with the content in advance and be prepared to perceive the whole lecture. However, again, not all students look through the material in advance, even if they are asked to do so. 
In online classes, the perception of the contents of lectures may become even a greater issue. In such cases, the problem is partially removed where students share their computer screens with the instructor. However, this requires some considerable preliminary training in using digital technologies for both parties.

Working on term papers can be made remotely, partly as video conferences; at that, the work should follow the pattern of a practical class, and, partly, as an individual online class which requires a higher level of digital experience of both the instructor and the student, and it's also time consuming.

From here we may conclude that for high-quality online training, it is essential that the curricula first include subjects related to training in various digital technologies, and only then there are special subjects related to the chosen specialty. In addition, every instructor should regularly go through an advanced training course in digital technologies, since they progress too fast in the world today.

As regards intermediate testing of students in certain sciences, this can be performed remotely in two ways: testing based on the electronic information and educational environment of the relevant university, and also as a video conference mode or by a video call in a messenger. The disadvantage of testing is the lack of control of the students by the instructor throughout the test. The disadvantages of video interchange may be the inability to save the visual results of the intermediate test (traces of a video conversation can only be saved in a video format, which will obviously be hard for perception by an external supervisor), as well as little time (or no time at all) for a student to prepare their answers.

\section{Discussion}

A significant element of learning is to maintain feedback from the student to the instructor. With the total distance learning, this connection gets nearly lost, since the student does not meet with the instructor even at tests. In the video conference mode, you can answer some of the students' questions, but such feedback is severely compromised due to the lack of visual contact. The similarity of this contact could be provided by conducting a video call conversation in a messenger, which was performed by some instructors of the Don State Technical University. With this method of providing feedback, at least three drawbacks are identified: the instructor spends a lot of time on individual conversations, while the other students do not hear the main part of the conversation and the learning element is not even brought to them indirectly; the student can read information from other devices during a video call conversation, and the instructor won't fully assess the student's knowledge of the issue discussed.

Among the positive effects of restrictive measures caused by to the spread of the COVID -19, we should note the rapid development of online learning technologies by instructors, and, as many instructors claim, an increased attendance at online classes compared to offline ones. Even those instructors who did not initially rush to create online courses were now forced to do so, because they were challenged with a choice to create such courses and master the appropriate digital technologies, or to stop teaching at all. Students who combined their studies with work were now able to attend online classes due to the ease of connection and the lack of need to spend time to get to the place of study.

It is noteworthy that the total switchover of the whole body of students to online learning in the spring of 2020 still revealed more shortcomings than positive aspects. In addition to the psychological and psycho-physiological problems discussed above, the quality of training has suffered. It was revealed that not all types of classes and not all sciences can be taught online without a significant loss in the effectiveness of learning. If 
the disadvantage of the remote mode is only the lack of the effect of live communication, then to instill in students the necessary skills and abilities in online classes was only for those instructors who were digitally educated, who, as a rule, taught subjects related to the development of computer technologies. Laboratory classes and training practices turned out to be completely impossible to conduct.

Because of the deteriorating quality of education and the actual transfer of students from full-time to part-time format, the students of a few universities who are studying on a compensatory form of education and have paid for full-time education demanded a refund of their fees, since the part-time format is usually five times cheaper than that of full-time.

Many schools have stumbled upon the problem of using copyrights and related rights to the information technologies applicable for distance education, with the technical unavailability of the university material base to ensure sustainable online work due to the increased load on services that were previously used as teaching aids. For example, The SKIF platform at the Don State Technical University has failed to provide a high-quality support in the mass mode of distance learning: low quality of the provided signal, which led to bad sound, periodic lack of access to the platform for some students, and other shortcomings. This was further complicated by some general problems with communication which were aggravated in some remote areas due to the low performance of their telecommunications networks. It should be understood that many students who were forced to move from university dormitories to other accomodations, also in hard-to-reach areas, during the period of restrictions caused by the COVID-19, were almost completely deprived of the opportunity to attend online classes through digital communication channels.

On switching to the online format, the socially educational activities at the university are suffering greatly. Neither the curators nor the instructors can provide their educational influence in an online video class. More than that, of course, a significant part of educational work is carried out in the classroom, where the instructor turns into an educator, i.e. not only shares knowledge, but also educates students, introducing them to the proper code of conduct and culture practices, etc. Socially educational work, in addition, where introduced correctly, is performed comprehensively, i.e. students get involved in the scientific and methodological work of a corresponding department. The correct approach to educational work allows to pick out students who want to engage in research, identify young talents and improve academic performance in regular classes and supervisor student meetings. However, all this is impossible in the context of distance learning.

The nearly six-month experience of the instructors of the Don State Technical University in teaching students remotely has revealed the following types of interaction, the digitalization of which seems most promising.

Summing up, as recommendations for improving educational technologies in the context of digitalization, the authors offer:

1. Initially, when developing training based on individual plans, it is the online learning that takes over. Therefore, with this mode of training, digitalization should cover nearly the entire learning process, and meetings with instructors and scientific supervisors in full-time format can be made periodically just to clarify some most difficult issues of mastering subjects and current research, as well as (partially) to conduct intermediate testing in sciences, practical works and term projects.

It is possible to partially computerize the training of students in groups based on regular curricula. First of all, the experience of the Don State Technical University has shown that digital technologies are most successfully used at performing the final state examination. The presentation of the diploma works was successfully conducted at video conferences. As 
it turned out, this is no more difficult to organize than a normal shop meeting held remotely. Failures in the operation of the equipment during the state final examination both in June and December 2020 were rare. In only one per cent of all cases, it was necessary to postpone the presentation of a student's diploma work due to the malfunctioning communication equipment they were using to communicate with the examination board. Undoubtedly, the organization of the presentation of final diploma works requires a highly qualified secretary of the board. The secretary, a few days before the presentation of the final diploma, must properly check the performance of the student's equipment and their ability to use the relating software. To help make the work of the secretary easier, such checking can be done by the supervisors of the students' final diploma works.

In the totally digital format, it is possible to deliver lectures in humanities, technical and natural sciences; it is possible to partially handout lecture contents in digital format. During the lectures at video conferences at the Don State Technical University, students had trouble perceiving formulas and doing geometric tracing. To solve this problem, instructors are bound to send electronic handouts to students beforehand to make them get familiarized with the content in advance and be prepared to perceive the whole lecture. However, again, not all students look through the material in advance, even if they are asked to do so.

In online classes, the perception of the contents of lectures may become even a greater issue. In such cases, the problem is partially removed where students share their computer screens with the instructor. However, this requires some considerable preliminary training in using digital technologies for both parties.

Working on term papers can be made remotely, partly as video conferences; at that, the work should follow the pattern of a practical class, and, partly, as an individual online class which requires a higher level of digital experience of both the instructor and the student, and it's also time consuming.

From here we may conclude that for high-quality online training, it is essential that the curricula first include subjects related to training in various digital technologies, and only then there are special subjects related to the chosen specialty. In addition, every instructor should regularly go through an advanced training course in digital technologies, since they progress too fast in the world today.

As regards intermediate testing of students in certain sciences, this can be performed remotely in two ways: testing based on the electronic information and educational environment of the relevant university, and also as a video conference mode or by a video call in a messenger. The disadvantage of testing is the lack of control of the students by the instructor throughout the test. The disadvantages of video interchange may be the inability to save the visual results of the intermediate test (traces of a video conversation can only be saved in a video format, which will obviously be hard for perception by an external supervisor), as well as little time (or no time at all) for a student to prepare their answers. When being tested in a specially equipped room (e.g. in case of the Unified State Exam), the students are subject to control from some authorized persons. Another thing is when a student has the opportunity to do a test from any device by logging in to a special program. The experience of testing during the summer session of the 2019-2020 academic year and the winter session of the 2020-2021 academic year showed that unscrupulous students, taking the test, searched the answers to theoretical questions in the Internet, while the conditions of the problems were saved for finding their solutions later, thus doing a number of attempts to pass the test, they had a readymade solution at the next attempt. Moreover, in the absence of proper control, some companies may profit from offering and assisting students conducting intermediate testing. At regular times, such mediators earn money making term projects, various calculations, graphics and test works, as well as final 
graduation theses. At distance learning caused by some restrictive measures due to the spread of the COVID - 19, there have been detected a number of appeals to such "entrepreneurs" from students who resorted to cheating at going through tests in certain subjects.

Therefore, it seems quite logical to use a combined approach to conducting intermediate testing in subjects. Of course, it should be highly desirable to have the results of the taken test as a documentary confirmation of the development of the student's knowledge, skills and mastery they acquired while learning the subject. However, the instructor must have the right to additionally control the student's knowledge and skills orally, e.g. as an interview on the issues of the test passed by the student. If the university uses a blended online and offline learning system, then the student must answer the teacher's clarifying questions after passing the test at a person-to-person meeting. If it is impossible to organize such a face-toface meeting (e.g. the student is staying in another locality according to an individual study plan, or there are legally established restrictions on face-to-face meetings, as during a pandemic), then a video interview or video call may serve as substitutes. This arrangement of intermediate testing calls for the maximum use of computer technologies. With this blended approach, it seems logical to lower the grade obtained during the test, based on the results of the interview. However, there should not be a reverse situation (an increase in the grade), so that the result of the objective assessment of the student's knowledge, skills and mastery, set by the testing program, is not emasculated.

\section{Conclusion}

Considering the above, there should be no doubt that the restrictive measures imposed by the Ministry of Science and Higher Education of Russia, as well as the authorities of most of the constituent entities of the Russian Federation, have contributed to the accelerated progress of computer technologies in education, as well as the rapid adoption of these technologies by the staff and students. In the future, it should be vital to increase the share of digital technologies in education, promote the study of computer-related sciences at any professional training. Digitalization should not lead to the abolition of full-time education, but, instead, should be used in every form: full-time, part-time and distance learning.

\section{References}

1. N.J. Albury, Crit. Inq. Lang. Stud. 12(4), 256-282 (2015). doi:10.1080/15427587.2015.1096732

2. D.S. Anderson. Lang. Educ. 25 (4), 273-289 (2011). doi:10.1080/09500782.2011.577218

3. M.De Meulder, A. Kusters, E. Moriarty, J.J. Murray, Multicult. Dev. 2 (2), 1-15 (2019). doi:10.1080/01434632.2019.1592181

4. P. Garrett, Cambridge Univ. Press, Cambridge, UK (2011). doi:10.1017/CBO9780511844713

5. Zoltán Kövecses, Review of Cognitive Linguistics 18(1), 112-130 (2020). doi:10.1075/rcl.00053.kov

6. Richard Trim, Metaphor and the Historical Evolution of Conceptual Mapping (NY: Palgrave Macmillan, New York, 2011).

7. Fan, Procedia Computer Science 131, 925-928, (2018). doi.org/10.1016/j.procs.2018.04.224.

8. Safar J. Appl. Ling. Rev. (2017). doi: 10.1515/applirev-2017-0082

9. J. Safar, O. Le Guen, G. Collí Collí, Hau M. Collí, Sign Lang. Stud. 18 (4), 488-516 (2018). doi:10.1353/sls.2018.0014

10. Sallabank J. Multilingua 38 (2018). doi: 10.1515/multi-2018-0002 
11. T.B. Mikheeva, E.V. Murugova, SHS Web of Conf (2019). doi.org/10.1051/shsconf/20197007003

12. S.V. Pervukhina, O.A. Evtouchenko, L.Iu. Kotliarenko, M.P. Churikov, SHS Web of Conferences. Curr. Iss. Mod. Ling. Hum. 232-244 (2020). doi: 10.1051/shsconf/20208801013

13. M.A. Tamarkin, A.N. Isaev, E.V. Murugova, V.I. Butenko, MATEC Web of Conf. (2018). doi:10.1051/matecconf/201822601025

14. S. Pervukhina, V. Demchenko, E3S Web of Conf. (ITSE 2020). 18033, doi:10.1051/e3sconf/202021018033

15. N.B. Boeva-Omelechko, M.R. Zheltukhina, O.P. Ryabko, G.G. Matveeva, E.V. Murugova, I.A. Zyubina, Space and Culture, India 4, 112-121 (2018). doi: 10.20896/saci.v6i4.387 